\title{
Perioperative Anesthetic Considerations for a Child with Mabry Syndrome
}

\section{Muellerleile PR* and Vustar M \\ Cardinal Glennon Children's Hospital, Saint Louis University, Saint Louis, USA}

*Corresponding author: Muellerleile PR, Cardinal Glennon Children's Hospital, Saint Louis University, Saint Louis, MO, 1465 S Grand Blvd, Saint Louis, MO 63104, USA, Tel: 6166380832; Email: Philip.Muellerleile@Health.slu.edu

\section{Case Report \\ Volume 3 Issue 1}

Received Date: December 04, 2017

Published Date: January 09, 2018

\section{Abstract}

Objective: Mabry Syndrome is a rare genetic disorder with a constellation of anatomic and physiologic abnormalities. We present the case report of a patient with Mabry syndrome who has undergone an extensive variety of anesthetic encounters.

Case Report: 4 year old girl with Mabry syndrome who exhibits classic anatomical abnormalities such as facial dysmorphism, brachytelephalangy, and hypotonia, as well as physiologic and cognitive abnormalities such as hyerphosphatasia, seizure disorder, and cognitive delay.

Conclusion: Despite facial anatomic abnormalities, hyperphosphatasia, and hypotonia, traditional anesthetic agents/techniques were successful in this Mabry patient who has undergone greater than twenty anesthetic encounters.

Keywords: Mabry Syndrome; Hyperphosphatasia; Facial Dysmorphism; Hypotonia

Abbreviations: HPIDS: Hyperphosphatasiaintellectual disability syndrome; GPI: Glycosyl Phosphatidy Linositol

\section{Introduction}

Mabry syndrome or hyperphosphatasia-intellectual disability syndrome (HPIDS) is a constellation of cognitive, neurologic, and anatomic abnormalities [1,2] resulting from a glycosylphosphatidylinositol (GPI) anchor deficiency [3]. GPI anchors attach to the extracellular surface of cell membranes, acting as a binding site for molecules such as alkaline phosphatase [3]. There are fewer than 30 documented cases of HPIDS, however prevalence is unknown. Clinical signs include facial dysmorphism [4], brachytelephalangy [4], seizures
[1], CNS structural abnormalities, hypotonia, Hirschsprung's disease, and a varying degree of intellectual/cognitive delay [2]. Laboratory findings include hyperphosphatasia up to 20 times the upper reference range [4], with some individual exhibiting intracellular inclusions $[1,3,4]$.

\section{Case Study}

We present a 4 year old child with HPIDS who has undergone greater than 20 anesthetic events. This patient has a complex medical history which includes cerebral palsy, developmental delay, dysmorphic features (facial as well as hemihypertrophy of a lower extremity), hypotonia, and seizures (grand mal). At two weeks of age the patient presented with multifocal bowel ischemia 


\section{Anaesthesia \& Critical Care Medicine Journal}

resulting in multiple bowel surgeries/resections and development of short gut syndrome, with total parenteral nutrition dependence. Surgical history consists of abdominal/bowel surgeries, gastrointestinal procedures, and vascular access procedures.

Induction of anesthesia for the procedures performed utilized propofol or ketamine, and sevoflurane for anesthetic maintenance, without complications. The majority of the procedures utilized vecuronium or rocuronium for neuromuscular blockade. Succinylcholine was administered during rapid sequence induction on two separate occasions. In the setting of hypotonia, neither prolonged muscular blockade nor hyperkalemia were observed. Despite facial dysmorphia, intubations with age appropriate endotracheal tubes were successful under direct visualization. Pain management was achieved through the use of fentanyl and morphine, without sensitivity or complications.

\section{Conclusion}

HPIDS is a rare autosomal recessive disorder [2,5] requiring specific anesthetic considerations. Despite facial feature involvement, including congenital laryngeal cartilage softening [5], airway variations leading to difficult intubation have not been documented, and were not appreciated in this patient. Seizure history, isolated or reoccurring, is common in this population. Continuation of perioperative seizure prophylaxis with availability of sufficient seizure abortive options should be considered. HPIDS hypotonia did not appear to have an effect on either depolarizing or nondepolarizing neuromuscular blocking agents. Furthermore, in the setting of GPI deficiency, neither pharmacokinetic nor pharmacodynamic abnormalities were appreciated during administration of the aforementioned anesthetic or pain management agents.

\section{References}

1. Mabry CD, Bautista A, Kirk Richard FH, Dubilier LD, Braunstein H, et al. (1970) Familial hyperphosphatasia with mental retardation, seizures, and neurologic deficits. Journal of Pediatrics 77(1): 74-85.

2. Thompso M, Roscioli T, Marcelis C, Nezarati MM, Stolte Dijkstra I, et al. (2012) Phenotypic variability in hyperphosphatasia with seizures and neurologic deficit (Mabry Syndrome). American Journal of Medical Genetics 158A(3): 553-558.

3. Cole D, Thompson M (2015) Neurogenetic aspects of hyperphosphatasia in Mabry Syndrome. Subcell Biochem 76: 343-361.

4. Thompson M, Nezarati MM, Gillessen Kaesbach G, Meinecke P, Mendoza Londono R, et al. (2011) Hyperphosphatasia with seizures, neurologic deficit, and characteristic facial features: Five new patients with Mabry syndrome. Am J Med Genet A 155A(7): 1661-1669.

5. Xue J, Li H, Zhang Y, Yang Z (2016) Clinical and genetic analysis of two Chinese infants with Mabry syndrome. Brain Dev 38(9): 807-818.

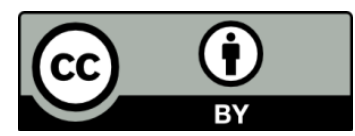

